\title{
Determining optimal population rates of cardiac catheterization: A phantom alternative?
}

\author{
Madhu K. Natarajan, Amiram Gafni, Salim Yusuf \\ § See related article page 35
}

$\mathrm{C}$ ardiac catheterization is a key step in the diagnosis and management of coronary artery disease (CAD). Recent advances in percutaneous coronary interventions (PCI) and coronary artery bypass grafting (CABG) have enhanced options for the management of patients and have led to considerable expansion of the availability of cardiac catheterization across Canada and elsewhere. Whether our catheterization rates are appropriate has been debated for almost 2 decades. In 1988/89, the average rate in Canada was 236 per 100000 (range 194 in the Atlantic provinces to 280 in Alberta). ${ }^{1} \mathrm{~A}$ recent report has shown much higher rates across the country (e.g., 500 per 100000 in Ontario in 2001/02) that are comparable to many in European countries but are still considerably lower than rates in the United States., ${ }^{2,3}$ The question remains: Are Canadian catheterization rates optimal, too low or too high?

In this issue (page 35), Graham and colleagues ${ }^{4}$ attempt to determine the optimal population rate for cardiac catheterization. Using data collected between 1995 and 2002 from the Alberta Provincial Project for Outcome Assessment in Coronary Heart Disease (APPROACH) registry, they found that the age-adjusted average catheterization rates varied by region and sex (range 404.9 to 638.1 per 100000 population aged over 20 years among men and 171.8 to 314.0 per 100000 among women). Further, the number of patients found to have high-risk CAD increased linearly with increasing catheterization rates, without any evidence of a plateau in the number. The authors argue that these data indicate that a much higher rate of catheterization procedures may be required across Canada.

In this commentary we consider whether defining and measuring an optimal population rate for catheterization is possible and, if so, whether the optimal rate provides useful information for decision-makers or is only a "phantom alternative." We then discuss alternative conceptual directions to improve the system.

\section{Determining an optimal catheterization rate}

In trying to define the optimal rate of cardiac catheterization, one needs to define what one wants to optimize. In a health care system without budgetary constraints whose goal is to maximize the health of the overall population, one would like to perform catheterization procedures on all potential patients for whom the benefits outweigh the harms. Benefits might include reduced mortality or morbidity, relief of symptoms or simple reassurance, while harms might include increased mortality or morbidity associated with catheterization or with subsequent procedures (e.g., PCI or CABG), complications such as bleeding, and anxiety in patients (e.g., from waiting for the procedure or from simply being informed of a diagnosis of heart disease). These considerations mean that, at any given time, the optimal rate in a given population will be determined by the prevalence and incidence of CAD in that population, by the existing evidence for the value of how catheterization can improve management, and by its potential complications. However, even in this ideal situation (e.g., no constraints on resources) the optimal catheterization rates may differ between regions because of population differences (e.g., in age and sex distribution, and in risk-factor profile).

In reality, whatever resources are available are insufficient to support all effective interventions for all potential patients. As a result, we must choose between different ways of using the available resources with the goal of maximizing the overall health of the population. Resources are used efficiently if, and only if, the value of what is being gained from the use of more resources for catheterization exceeds the value of what is forgone by not using resources in alternative ways (e.g., to treat other diseases such as hypertension or cancer, or to fund other worthwhile non-health-related needs such as education or transportation)..$^{5}$

As it was in the ideal situation of unlimited resources, the optimal allocation of limited resources is also context specific. ${ }^{6}$ For example, consider the situation of 2 health care systems (A and B) with identical populations, but system $A$ is much richer than system $B$. It is easy to see that, if all else is equal, the optimal population rate of catheterization in system A would be greater than the rate in system B. Further, the optimal rate would depend on the profile of medical needs in the community. For example, if sys- 
tems A and B instead have similar overall resources for health care and have a similar prevalence and incidence of $\mathrm{CAD}$, but system $\mathrm{B}$ has a much higher rate of another disease (e.g., cancer), it would be reasonable to expect that each population would have a different optimal catheterization rate. Thus, differences in catheterization rates between 2 countries (or provinces or regions) reflect, among other things, differences in the absolute and relative population rates of various medical conditions (not just CAD), in societal values or preferences and in the availability of resources for health care.

\section{Optimal rate or "phantom alternative"?}

The analysis by Graham and coauthors in this issue $e^{4}$ does not help in determining the optimal population rate of catheterization, even for a health care system without resource constraints. Important limitations include a lack of estimate of the true prevalence of high-risk CAD in the population studied and little insight into why catheterization rates varied significantly between health regions in Alberta. Although Graham and coauthors point out that significantly higher rates of catheterization in the United States than in Canada have led to a 2- to 3fold increase in the number of people with left main disease being treated, others have reported that this strategy leads to an 8 to 9 -fold increase in surgery rates among patients with limited CAD, for whom the tradeoff between relative benefits and harms may be marginal. ${ }^{7}$ Further, the number of patients with complications such as disabling stroke or life-threatening bleeds following catheterization may increase, and thus increasing the catheterization rates may not lead to overall net benefits.

If the primary benefit of catheterization is to identify high-risk $\mathrm{CAD}$ to select patients for revascularization, an increase in catheterization rates without appropriate selection of patients referred for the procedure is likely to represent a poor use of scarce resources. Perhaps an alternative approach would be to develop strategies to select only patients who have a high pretest probability of having high-risk $\mathrm{CAD}$ and who would therefore derive the greatest benefit from catheterization. Unfortunately, although formal and objective models are available, they are used rarely in clinical practice. ${ }^{8}$ A commonly cited reason is that these indices have limited clinical utility in deciding on treatment strategies at the individual clinicianpatient interaction level. However, in a recent analysis of a large number of baseline variables on the catheterization referral forms in Hamilton, Ont., the urgency ranking of the patient by the referring physician (urgent v. elective status) did not predict major adverse events during the waiting period nor high-risk CAD on coronary angiography (unpublished data). Therefore, although available objective criteria are not perfect predictors, they cannot be ignored.

Further, although the general assumption is that increased identification of high-risk CAD with increased catheterization rates will lead to long-term survival benefits, the data in support of this are contradictory. Several registries have not shown any survival advantage for patients with acute coronary syndrome admitted to hospitals with catheterization facilities (with markedly higher catheterization rates) compared with patients admitted to hospitals without such facilities (with markedly lower procedure rates) during both short- and longer-term (1-2 years) follow-up. ${ }^{9-11}$ Rates of hospital readmission because of unstable angina are lower with more aggressive catheterization, but this strategy does not necessarily represent efficient use of resources if a proportion of the procedures could have been avoided. These data are also consistent with a meta-analysis of randomized controlled trials of routine catheterization versus catheterization of selective cases of ischemia among patients with acute coronary syndromes that demonstrated no differences in mortality, a small reduction in myocardial infarction but larger differences in rates of hospital readmission because of unstable angina. ${ }^{12}$ Therefore, an increase in catheterization rates will no doubt increase the number of patients with high-risk CAD being identified, but it is unclear whether this will lead to improved survival.

What is clear is that the optimal rate for any procedure or medical intervention is contextual (e.g., prevalence and incidence of the disease, net benefits of the procedure, net benefits of procedures to treat other diseases and total budget available). The complexity of determining an optimal rate reflects the multiple facets of the question being addressed. Furthermore, the data required to determine the optimal catheterization rate are not currently available, even for the case of an unconstrained health care system. Thus, although decision-makers might be pressed to fund an optimal catheterization rate over other alternatives, optimizing the rate is in fact a phantom alternative. Phantom alternatives are a well-known phenomenon in decision science: ${ }^{13}$ they represent an illusory choice - one that may look real but that is unavailable at the time a decision is made. 


\section{Where do we go from here?}

A more productive alternative is to change the objective from optimization to unambiguous improvement of the effectiveness and efficiency of the catheterization system (Box 1). By finding ways that will continually improve the system, one will gradually get closer to the optimal rate of catheterization in the population that one serves. An important first step would be to act on what we already know. For example, a recent analysis indicated that, despite universal health care and increases in the availability of catheterization, there were significant inequities in access to catheterization and CABG after myocardial infarction within regions of Canada related to the socioeconomic status and sex of the patient and the specialty of the attending physician. ${ }^{14}$ If such inequities are measurable in populations of hospitalized patients, they are likely magnified several fold in outpatient populations of referrals for catheterization, which are initiated in thousands of primary care practices and specialist offices across the country. Improved efficiency of the referral process for outpatients might be achieved if standardized methods were developed, validated and adopted for screening and referring people at high risk of CAD for further investigations and possible catheterization; a greater emphasis on objective criteria would potentially reduce unjustifiable variability in access, provide more rapid access for patients who are likely to gain maximal benefit, and potentially reduce the size of waiting lists and the morbidity and mortality associated with waiting. ${ }^{2}$

Efficiency might also be improved if the available technology were used more wisely. Between $15 \%$ and $30 \%$ of patients undergoing catheterization have a normal or near normal angiogram..$^{15,16}$ Can we use our existing resources better (i.e., increase efficiency) to avoid catheterization in a proportion of these patients without compromising our ability to detect those with significant and correctable coronary anatomy and without compromising other benefits? Recent advances in noninvasive angiography (e.g.,

Box 1: Approaches to the efficient use of cardiac catheterization and other diagnostic tests

- State the objective(s) of the test

- Compare the benefits and the harms

- Develop objective criteria for patient referral to maximize the benefits and minimize the harms

- Incorporate and standardize these referral criteria for use in all practices, from primary care to specialist

- Audit referral patterns and utilization of the test, with regular feedback

- Act on the audit results (e.g., if referral patterns are inappropriate, discuss means of rectifying them)

- Incorporate new evidence, new diagnostic procedures (e.g., multi-slice CT angiography) into the referral process multi-slice CT angiography), which assesses the severity of $\mathrm{CAD}$, could divert a large number of patients with lowrisk CAD away from catheterization. However, widespread use of these procedures may paradoxically increase the number of referrals for catheterization (e.g., in order to validate a moderate narrowing of a coronary artery found on CT angiography) in relatively asymptomatic patients. Similarly, nonselective referral of patients for noninvasive cardiac testing (e.g., echocardiography, nuclear stress test) may lead to inconclusive or false-positive results, which may also serve as a source of inappropriate catheterization referrals. Standardized referral criteria for and after noninvasive tests that use formal Bayesian models, together with periodic audits of referral patterns and utilization, would help to increase the effectiveness of these tests. Finally, a realization that inappropriate referral of patients with little or minor CAD will divert resources from other health needs and also lead to some harm (e.g., increased risk of complications with little chance of benefit, patient anxiety) should lead to some "tempering" of many physicians' and patients' expectations (that all procedures should be done in all patients). This could help to set some internal limits within the practice styles of individual physician and health care systems.

Graham and coauthors have done us a service by reopening the discussion on optimal catheterization rates. ${ }^{17}$ However, because it is unlikely that the optimal rate for any given population can be calculated from available data, our focus should be primarily on improving efficiencies within our current system and viewing rates of catheterization, or for that matter any procedure, not in isolation but instead in the context of the overall needs of the population. ${ }^{6,18}$

This article has been peer reviewed.

From the Population Health Research Institute and Department of Clinical Epidemiology and Biostatistics, McMaster University, Hamilton, Ont.

Competing interests: None declared.

Contributors: All authors contributed equally to the concept and writing of the manuscript and provided final approval of the version to be published.

\section{References}

1. Higginson LAJ, Cairns JA, Keon WJ, Smith ER. Rates of cardiac catheterization, coronary angioplasty and open-heart surgery in adults in Canada. CMAF 1992;146(6):921-5.

2. Martin RM, Hemingway H, Gunnell D, Karsch KR, Baumback A, Frankel S. Population needs for coronary revascularization: Are national targets for England credible? Heart 2002;88:627-30.

3. Consensus Panel on Target Setting. Final report and recommendations. Report submitted to the Ontario Ministry of Health and Long-Term Care on March 15 , 2004. Toronto: Cardiac Care Network of Ontario; 2004. Available: www.ccn .on.ca/publications/Consensus_Panel_On_Target_Setting_FRR.pdf (accessed 2005 June 2).

4. Graham MM, Ghali WA, Faris PD, Galbraith PD, Tu JV, Norris CM, Zentner A, Knudtson ML; APPROACH Investigators. Population rates of cardiac catheterization and yield of high-risk coronary artery disease. CMAf 2005; 173(1):35-9.

5. Gafni A, Birch S. Inclusion of drugs in provincial drug benefit programs Should "reasonable decisions" lead to uncontrolled growth in expenditures? CMA7 2003;168(7):849-51. 
6. Birch S, Gafni A. Economics and the evaluation of health care programs: generalizability of methods and implications for generalizability of results. Health Policy 2003;64:207-19.

7. Tu JV, Naylor CD, Kumar D, DeBuono BA, McNeil BJ, Hannan EL. Coronary artery bypass graft surgery in Ontario and New York State. Which rate is right? Ann Intern Med 1997;126:13-9.

8. Antman EM, Cohen M, Bernink PJL, McCabe CH, Horacek T, Papuchis G et al. The TIMI risk score for unstable angina/non-ST elevation MI. A method for prognostication and therapeutic decision making. FAMA 2000;284: $835-42$.

9. Yusuf S, Flather M, Pogue J, Hunt D, Varigos J, Piegas L, et al. Variations between countries in invasive cardiac procedures and outcomes in patients with suspected unstable angina or myocardial infarction without initial ST elevation. OASIS (Organisation to Assess Strategies for Ischaemic Syndromes) Registry Investigators. Lancet 1998;352(9127):507-14.

10. Fox KA, Goodman SG, Klein W, Brieger D, Steg PG, Dabbous O, et al. Management of acute coronary syndromes. Variations in practice and outcome; findings from the Global Registry of Acute Coronary Events (GRACE). Eur Heart 7 2002;23(15):1177-89.

11. Van de Werf F, Gore JM, Avezum A, Gulba DC, Goodman SG, Budaj A, et al; GRACE Investigators. Access to catheterisation facilities in patients admitted with acute coronary syndrome: multinational registry study. $B M \mathcal{F}$ 2005;330(7489):441. Epub 2005 Jan 21

12. Mehta SR, Cannon CP, Fox KAA, Wallentin L, Boden WE, Spacek R, et al Routine versus selective invasive strategies in patients with acute coronary syndromes. A collaborative meta-analysis of randomized trials. $7 A M A$. In press.

13. Farquhar PH, Pratkanis ART: Decision structuring with phantom alternatives. Manage Sci 1993;39:1214-26.

14. Khaykin Y, Austin PC, Tu JV, Alter DA. Utilisation of coronary angiography after acute myocardial infarction in Ontario over time: Have referral patterns changes? Heart 2002;88:460-6.

15. Hemmelgarn BR, Ghali WA, Quan H, Brant R, Norris CM, Taub KJ, et al; APPROACH Investigators. Poor long-term survival after coronary angiography in patients with renal insufficiency. Am 7 Kidney Dis 2001;37(1):64-72.

16. Natarajan MK, Mehta SR, Holder DH, Goodhart DR, Gafni A, Shilton D, et al. The risks of waiting for cardiac catheterization: a prospective study [published errata CMA7 2003;168(2):152 and 2003;168(12):1529]. CMA7 2002; 167(11):1233-40.

17. Tu JV, Naylor CD, Pashos CL, McNeil BJ. Coronary angiography and revascularization after acute myocardial infarction: Which rate is right? Eur Heart 7 1998;19:529-30.

18. McGlynn EA. Perspective: There is no perfect health system. Health Aff 2004;23(3):100-2.

Correspondence to: Madhu K. Natarajan, Rm. 260, 2nd floor McMaster Clinic, Hamilton Health Sciences - General Site, 237 Barton St. E, Hamilton ON L8L 2X2;

natarajm@ccc.mcmaster.ca

\section{CMAJ.JAMCél}

CMAJ is powered by HighWire Press — the world leader in online journal publishing.

Search all of MEDLINE and access more than 340 highly cited journals, including CMAJ, the New England Journal of Medicine, JAMA and BMJ.

Visit cmaj.ca to sign up for:

- email table of contents service

- customized alerts on the topics of particular interest to you

- citation alerts on specific articles

\section{cmaj.ca}

